Original Contribution

\title{
MINERAL ELEMENTS AND BIOCHEMICAL ANALYSIS OF CALENDULA OFFICINALIS L. AFFECTED BY BIO-STIMULATORS
}

\author{
H. Rafiee ${ }^{1}$, A. Mehrafarin ${ }^{2}$, M. Labbafi ${ }^{2}$, A. Qaderi ${ }^{3}$, H. Naghdi Badi ${ }^{2 *}$ \\ ${ }^{1}$ Department of Horticulture, Science and Research Branch, Islamic Azad University, Tehran, Iran \\ ${ }^{2}$ Department of Cultivation and Development, Medicinal Plants Research Center, Institute of Medicinal \\ Plants, ACECR, Karaj, Iran \\ ${ }^{3}$ Department of Biotechnology, Medicinal Plants Research Center, Institute of Medicinal Plants, ACECR, \\ Karaj, Iran
}

\begin{abstract}
PURPOSE: The objectives were to evaluate the foliar application effects of bio-stimulators on morphological and phytochemical traits of pot marigold (Calendula officinalis L.). METHODS: This experiment was conducted on the base of randomized complete blocks design with three replications in two growth seasons of 2011 and 2012. The treatments included foliar application of commercial formulations of aminolforte, kadostim, fosnutren, and humiforte (each of them at 0.75 and 1.5 L.ha-1) based on amino acid compounds, $75 \mathrm{~kg}$.ha-1 chemical complete fertilizer (20:20:20\% of N:P:K), and control treatment (without bio-stimulators and fertilizer application). RESULTS: The bio-stimulators increased dry weight of capitula and leaves, total carbohydrates of leaves and capitula, and flavonoids of capitula in 2011, but they had not significant effect on these traits in respect of suitable ecological condition in 2012.

In 2011 and 2012, the most total dry weight (161.26 and 165.81 g.m. ${ }^{-2}$, respectively) and total flavonoids of leaves $\left(0.10\right.$ and $0.12 \%$, respectively) were obtained in $1.5{\mathrm{~L} . h a^{-1}}^{1}$ humiforte. In other way, the content of $\mathrm{N}, \mathrm{P}, \mathrm{K}, \mathrm{Fe}, \mathrm{Zn}, \mathrm{Cu}$, Mnand $\mathrm{Ca}$ was increased with application of bio-stimulators.

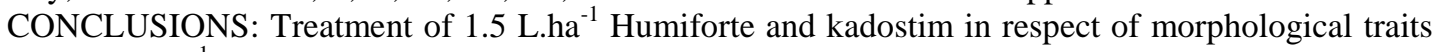
and $1.5 \mathrm{~L}_{\mathrm{L}} \mathrm{ha}^{-1}$ fosnutren, aminolforte and kadostim in regard to phytochemical traits were the best treatments which could be due to existence of amino acid compounds and macro-nutrients of $\mathrm{N}, \mathrm{P}$ and $\mathrm{K}$ in their formulations.
\end{abstract}

Key words: Calendula officinalis L., Bio-stimulators, Morphological and Phytochemical Traits

\section{INTRODUCTION}

Pot marigold (Calendula officinalis L.) from Compositae family is indigenous to central, eastern and southern Europe. It is cultivated commonly in North America, Balkans, Eastern Europe, Germany and India. This plant has a long history of usage as anti-inflammatory, antitumor, antioxidant, antibacterial, anti-HIV, anti-ulcer, antigenotoxic, chemoprotective and antiseptic properties (1). The Flavonoids compounds, one of pigments classes in $C$. officinalis, have antioxidant activities and they

* Correspondence to: Iranian Academic Center for Education, Culture and Research (ACECR), Institute of Medicinal Plants (IMP), Karaj, Iran.P.O.Box: 33651/66591, Tel: +98-263-4764010-9, Fax: +98263-4764021,Email: Naghdibadi@yahoo.com play an important role in human health by combating damage caused by oxidizing agents $(2,3)$.

Bio-stimulators as biological substances stimulate metabolism and metabolic processes to increase plants yield. These compounds such as commercial formulations of aminolforte, kadostim, fosnutren and humiforte have the basis of amino acid and they improve quantitative and qualitative growth (4). Micro and macro elements existing in bio-stimulators formulations play an important role in promoting the growth and production of plants. Micro elements participate in most of the enzymatic reactions and they also play a role indirectly through the synthesis of several 
growth regulators. The beneficial effects of these elements were reported by several authors, such as El-Kady, spraying $\mathrm{Zn}, \mathrm{Mn}, \mathrm{Cu}$ and $\mathrm{Fe}$ on sunflower plants (5, 6). Mandal et al. (2007) emphasized to increase of biochemical components in response to growth regulators on tea plants (7).

The objectives of this study were to investigate the foliar application effects of bio-stimulators on morphological and phytochemical traits of Calendula officinalis L.

\section{MATERIALS AND METHODS}

To investigate the effects of bio-stimulators on growth and phytochemical traits of Calendula officinalis L., a field experiment was conducted at Medicinal Plants Institute (MPI) in the Academic Centre for Education, Culture \& Research (ACECR), on the basis of randomized complete blocks design and three replications in two cropping seasons (during 2011 and 2012 years). The characterizations of field experiment and analysis of soil texture were as follows: $35^{\circ}$ $36^{\prime} \mathrm{N}$ and $50^{\circ} 56^{\prime} \mathrm{E}$; $1426 \mathrm{~m}$ elevation, loam-silty with $79 \%$ sand, $13 \%$ silt, $8 \%$ clay, EC 2.71 ds.m ${ }^{-1}, 0.071 \% \mathrm{~N}, 48.9 \mathrm{mg} . \mathrm{kg}^{-1}$ phosphorous, $33.6 \mathrm{mg} \cdot \mathrm{kg}^{-1}$ potassium, and $8.3 \mathrm{pH}$. In the experimental years, the average of rainfall received in the second year $(283 \mathrm{~mm})$ was higher than the first year $(210 \mathrm{~mm})$. Rainfall distribution to months was irregular in both years. Also, the average of relative humidity was 30 and 35\% in 2011 and 2012, respectively (45). The average of minimum and maximum temperatures in 2011 was 15 and $25^{\circ} \mathrm{C}$, while they were 12 and $21^{\circ} \mathrm{C}$ in 2012.
The treatments were foliar application of commercial formulations of aminolforte $\left(\mathrm{A}_{1}\right.$, and $\left.A_{2}\right)$, kadostim $\left(K_{1}\right.$, and $\left.K_{2}\right)$, fosnutren $\left(F_{1}\right.$, and $\left.\mathrm{F}_{2}\right)$, and humiforte $\left(\mathrm{H}_{1}\right.$, and $\left.\mathrm{H}_{2}\right)$ each of them at 0.75 and $1.5 \mathrm{~L}^{-\mathrm{ha}^{-1}}$ based on amino acid compounds, $75 \mathrm{~kg} \cdot \mathrm{ha}^{-1}$ pre-sowing of chemical complete fertilizer (CF) (20:20:20\% of N:P:K), and control treatment or without bio-stimulators and fertilizers application (C). The seeds were sown in rows $50 \mathrm{~cm}$ apart with inter-row spacing of $20 \mathrm{~cm}$ apart in the last week of April and after two weeks emergence occurred. Each experimental plot contained of 5 rows. The replications (blocks) with a distance of $1.5 \mathrm{~m}$ from each other and plots with a distance of $1 \mathrm{~m}$ from every side were considered. The seeds with proper quality of germination were supplied from the seed bank of the ACECR, Institute of Medicinal Plants. The irrigation and other field practices had been done as needed. To increase the absorption of solutions by plants, foliar application of bio-stimulators was done in conditions without wind and rain and before sunrise when plant stomata are open. Foliar application was done in 3 intervals every 15 days. First sample was collected 60 days after emergence. Samples in nylon bags were sent to laboratory for measuring parameters. Four commercial formulations of bio-stimulators including aminolforte, kadostim, fosnutren and humiforte were supplied by Inagrosa Industries Agro Biologicals, Madrid, Spain. The details of the formulations are mentioned in Table 1.

Table 1. Formulation of bio-stimulators used in the experimental treatments

\begin{tabular}{|c|c|}
\hline Biostimulators & Formulation of compounds ${ }^{* * *}$ \\
\hline $\begin{array}{l}\text { Aminolforte } \\
\text { (A) }\end{array}$ & $\begin{array}{l}3750 \mathrm{mg} . \mathrm{L}^{-1} \text { free amino acids, } 2 \% \text { organic components, and } 1.1 \% \text { total } \mathrm{N}(0.8 \% \text { urea } \mathrm{N} \text {, and } 0.3 \% \\
\text { organic } \mathrm{N})\end{array}$ \\
\hline Kadostim $(\mathrm{K})$ & $\begin{array}{l}3750 \mathrm{mg} . \mathrm{L}^{-1} \text { free amino acids, } 2 \% \text { organic components, } 5 \% \text { total } \mathrm{N}(0.9 \% \text { ammonia } \mathrm{N}, 3.4 \% \text { nitric } \\
\mathrm{N} \text {, and } 0.7 \% \text { organic } \mathrm{N}) \text {, and } 6 \% \text { potassium }\left(\mathrm{K}_{2} \mathrm{O}\right)\end{array}$ \\
\hline Humiforte $(\mathrm{H})$ & $\begin{array}{l}3750 \mathrm{mg} . \mathrm{L}^{-1} \text { free amino acids, } 2 \% \text { organic components, } 6 \% \text { total } \mathrm{N}(1.4 \% \text { ammonia } \mathrm{N}, 3.7 \% \text { urea } \\
\mathrm{N}, 0.5 \% \text { nitric } \mathrm{N} \text {, and } 0.4 \% \text { organic } \mathrm{N}), 5 \% \text { potassium }\left(\mathrm{K}_{2} \mathrm{O}\right) \text {, and } 3 \% \text { phosphorous }\left(\mathrm{P}_{2} \mathrm{O}_{5}\right)\end{array}$ \\
\hline Fosnutren $(\mathrm{F})$ & $\begin{array}{l}3750 \mathrm{mg} . \mathrm{L}^{-1} \text { free amino acids, } 2 \% \text { organic components, } 3.8 \% \text { total } \mathrm{N}(2.1 \% \text { ammonia } \mathrm{N}, 1.4 \% \\
\text { nitric } \mathrm{N} \text {, and } 0.3 \% \text { organic } \mathrm{N}) \text {, and } 6 \% \text { phosphorous }\left(\mathrm{P}_{2} \mathrm{O}_{5}\right)\end{array}$ \\
\hline
\end{tabular}


The measured parameters are as follows: capitula dry weight $\left(\mathrm{g} \cdot \mathrm{m}^{-2}\right)$, leaves dry weight $\left(\mathrm{g} . \mathrm{m}^{-2}\right)$, total dry weight $\left(\mathrm{g} . \mathrm{m}^{-2}\right)$, total carbohydrates of leaves and capitula (mg. $\mathrm{g}^{-1}$ DW), total flavonoids of leaves and capitula (\%), content of $\mathrm{N}(\%), \mathrm{P}$ (mg.g $\left.{ }^{-1} \mathrm{DW}\right), \mathrm{K}$ (mg. $\mathrm{g}^{-1}$ DW), Fe (mg. ${ }^{-1}$ DW), Zn (mg.g ${ }^{-1}$ DW), Cu (mg. $\left.\mathrm{g}^{-1} \mathrm{DW}\right)$, Mn (mg. $\left.\mathrm{g}^{-1} \mathrm{DW}\right)$, and $\mathrm{Ca}\left(\mathrm{mg} \cdot \mathrm{g}^{-1}\right.$ DW) in the leaves. The content of flavonoids in the examined plant extracts was determined using spectrophotometric method (8). The sample contained $1 \mathrm{ml}$ of methanol solution of the extract in the concentration of $1 \mathrm{mg} \cdot \mathrm{ml}^{-1}$ and $1 \mathrm{ml}$ of $2 \% \mathrm{AlCl} 3$ solution dissolved in methanol. The samples were incubated for an hour at room temperature. The absorbance was determined using spectrophotometer at $\lambda \max =$ $415 \mathrm{~nm}$. The samples were prepared in triplicate for each analysis and the mean value of absorbance was obtained. The same procedure was repeated for the standard solution of rutin and the calibration line was construed. Based on the measured absorbance, the concentration of flavonoids was read $\left(\mathrm{mg} \cdot \mathrm{ml}^{-1}\right)$ on the calibration line; then, the content of flavonoids in extracts was expressed in terms of rutin equivalent $(\mathrm{mg}$ of RU.g $\mathrm{g}^{-1}$ of extract) (9). Total soluble sugars were determined in the methanolic extract by using the phenol-sulphoric method according to Dubois et al., 1966 (10). Nitrogen, phosphorus, potassium and micro elements of $\mathrm{Fe}, \mathrm{Zn}, \mathrm{Cu}, \mathrm{Mn}$ and $\mathrm{Ca}$ were determined in dried leaves according to Wahing et al. 1989, Chapman and Pratt, 1961 and Garcia et al. 2009 (11, 12, 13). Combined analysis of the results for the growing seasons was done using the SPSS software (ver. 17), and means in the results were compared using the Fisher's protected Least Significant Differences (LSD) test.

\section{RESULTS}

According to the results of combined analysis of variance, the effect of year was significant on all of the morphological and phytochemical parameters and elements content of Calendula officinalis $\mathrm{L}$. The foliar application effect of biostimulators was significant on total dry weight, total flavonoids of leaves and $\mathrm{K}, \mathrm{Fe}$ and $\mathrm{Ca}$ content of leaves in both experimental years. Only in 2011 year, it was also significant on other parameters such as capitula and leaves dry weight, total flavonoids of capitula, total carbohydrate of leaves and capitula and elements contents of $\mathrm{N}, \mathrm{P}, \mathrm{Zn}, \mathrm{Cu}$ and $\mathrm{Mn}$ (Table 2).

Table 2. Combined Analysis of variance for effects of bio-stimulators on the measured parameters of pot marigold (Calendula officinalis L.) during 2011 and 2012 growth season

\begin{tabular}{|c|c|c|c|c|c|c|c|c|c|}
\hline \multirow{3}{*}{ S.O.V } & \multirow{3}{*}{ Years } & \multirow{3}{*}{$\mathrm{df}$} & \multicolumn{7}{|c|}{ Mean square } \\
\hline & & & \multirow{2}{*}{$\begin{array}{l}\text { Capitula } \\
\text { dry weight } \\
\left(\mathrm{g} \cdot \mathrm{m}^{-2}\right)\end{array}$} & \multirow{2}{*}{$\begin{array}{l}\text { Leaves } \\
\text { dry } \\
\text { weight } \\
\left(\mathrm{g} \cdot \mathrm{m}^{-2}\right)\end{array}$} & \multirow{2}{*}{$\begin{array}{l}\text { Total dry } \\
\text { weight } \\
\left(\mathrm{g} \cdot \mathrm{m}^{-2}\right)\end{array}$} & \multicolumn{2}{|c|}{$\begin{array}{l}\text { Total carbohydrates } \\
\left(\mathrm{mg} \cdot \mathrm{g}^{-1} \mathrm{DW}\right)\end{array}$} & \multicolumn{2}{|c|}{ Total flavonoids (\%) } \\
\hline & & & & & & Capitula & Leaves & Capitula & Leaves \\
\hline \multirow{3}{*}{ Block } & 2011 & 2 & 52.601 & 4.183 & 331.668 & 0.001 & 0.001 & 0.001 & 0.0002 \\
\hline & 2012 & 2 & 144.477 & 27.076 & 600.481 & 0.009 & 0.013 & 0.009 & 0.0003 \\
\hline & Means & 2 & 39.644 & 24.219 & 112.067 & 0.009 & 0.010 & 0.004 & 0.0003 \\
\hline Year (Y) & - & 1 & $205.054^{*}$ & $23.89^{*}$ & $3209.676^{* *}$ & $0.576^{* *}$ & $0.024^{* *}$ & $1.350^{* *}$ & $0.011^{* *}$ \\
\hline \multirow{3}{*}{$\begin{array}{l}\text { Treatment } \\
\text { (T) }\end{array}$} & 2011 & 9 & $126.952^{*}$ & $12.97^{* *}$ & $1145.507^{*}$ & $0.001^{* *}$ & $0.001^{* *}$ & $0.005^{* *}$ & $0.001^{\text {** }}$ \\
\hline & 2012 & 9 & $31.363^{\mathrm{ns}}$ & $5.831^{\mathrm{ns}}$ & $1514.104^{* *}$ & $0.001^{\mathrm{ns}}$ & $0.0003^{\text {ns }}$ & $0.001^{\mathrm{ns}}$ & $0.0004^{* *}$ \\
\hline & Means & 9 & $142.135^{* *}$ & $11.998^{*}$ & $2275.630^{* *}$ & $0.002^{*}$ & $0.002^{\mathrm{ns}}$ & $0.006^{* *}$ & $0.001^{* *}$ \\
\hline \multirow{2}{*}{$\begin{array}{l}\text { Year } \times \text { Treatm } \\
\text { ent }\end{array}$} & - & 9 & $16.180^{\mathrm{ns}}$ & $6.805^{\text {ns }}$ & $383.981^{\mathrm{ns}}$ & $0.00041^{\mathrm{ns}}$ & $0.0001^{\text {ns }}$ & $0.001^{\mathrm{ns}}$ & $0.0003^{\mathrm{ns}}$ \\
\hline & 2011 & 18 & 48.354 & 2.827 & 330.102 & 0.0001 & 0.002 & 0.001 & 0.0001 \\
\hline \multirow[t]{3}{*}{ Error } & 2012 & 18 & 20.140 & 5.945 & 197.186 & 0.001 & 0.001 & 0.001 & 0.00007 \\
\hline & Means & 38 & 40.731 & 4.526 & 292.930 & 0.001 & 0.001 & 0.001 & 0.0001 \\
\hline & 2011 & & 19.11 & 7.64 & 13.93 & 4.16 & 8.31 & 15.27 & 14.28 \\
\hline \multirow[t]{2}{*}{$\mathrm{CV}(\%)$} & 2012 & & 11.19 & 10.48 & 9.68 & 7.18 & 15.05 & 6.23 & 7.96 \\
\hline & Means & & 16.69 & 9.40 & 12.42 & 9.30 & 16.64 & 9.03 & 10.98 \\
\hline
\end{tabular}

In each column, ${ }^{*}, *$, and ${ }^{\mathrm{ns}}$ means significant at $0.01,0.05$ probability level, and non-significant, respectively. 
Table 2. continued

RAFIEE H., et al.

\begin{tabular}{|c|c|c|c|c|c|c|c|c|c|c|}
\hline \multirow[b]{2}{*}{ S.O.V } & \multirow[b]{2}{*}{ Years } & \multirow[b]{2}{*}{$\mathrm{df}$} & \multicolumn{8}{|c|}{ Mean square } \\
\hline & & & $\mathrm{N}(\%)$ & $\begin{array}{c}\mathrm{P} \\
\text { (mg.g } \\
\text { DW) }\end{array}$ & $\begin{array}{c}\mathrm{K}\left(\mathrm{mg} \cdot \mathrm{g}^{-1}\right. \\
\mathrm{DW})\end{array}$ & $\begin{array}{c}\mathrm{Fe}\left(\mathrm{mg} \cdot \mathrm{g}^{-}\right. \\
\left.{ }^{1} \mathrm{DW}\right)\end{array}$ & $\begin{array}{c}\mathrm{Zn} \\
\left(\mathrm{mg} . \mathrm{g}^{-1}\right. \\
\mathrm{DW})\end{array}$ & $\begin{array}{c}\mathrm{Cu} \\
\left(\mathrm{mg} . \mathrm{g}^{1}\right. \\
\mathrm{DW})\end{array}$ & $\begin{array}{c}\mathrm{Mn} \\
\left(\mathrm{mg} \cdot \mathrm{g}^{-1}\right. \\
\mathrm{DW})\end{array}$ & $\begin{array}{c}\mathrm{Ca} \\
\left(\mathrm{mg} \cdot \mathrm{g}^{-1}\right. \\
\mathrm{DW})\end{array}$ \\
\hline \multirow[t]{3}{*}{ Block } & 2011 & 2 & 0.638 & 0.045 & 0.329 & 0.078 & 0.145 & 0.007 & 0.119 & 15.82 \\
\hline & 2012 & 2 & 0.002 & 0.103 & 0.940 & 0.154 & 0.057 & 0.012 & 0.155 & 19.27 \\
\hline & Means & 2 & 0.283 & 0.135 & 1.190 & 0.223 & 0.192 & 0.018 & 0.273 & 34.91 \\
\hline Year (Y) & - & 1 & $1.329^{* *}$ & $18.08^{* *}$ & $72.23^{* *}$ & $1.072^{* *}$ & $0.077^{* *}$ & $0.121^{* *}$ & $21.62^{* *}$ & $72.42^{* *}$ \\
\hline \multirow{3}{*}{$\begin{array}{l}\text { Treatment } \\
\text { (T) }\end{array}$} & 2011 & 9 & $0.126^{* *}$ & $0.028^{* *}$ & $0.464^{* *}$ & $0.203^{* *}$ & $0.019^{* *}$ & $0.003^{* *}$ & $0.052^{* *}$ & $64.22^{* *}$ \\
\hline & 2012 & 9 & $0.031^{\mathrm{ns}}$ & $0.007^{\mathrm{ns}}$ & $6.913^{* *}$ & $0.088^{*}$ & $0.005^{\mathrm{ns}}$ & $0.001^{\mathrm{ns}}$ & $0.013^{\mathrm{ns}}$ & $16.058^{* *}$ \\
\hline & Means & 9 & $0.142^{*}$ & $0.031^{\mathrm{ns}}$ & $3.765^{* *}$ & $0.252^{* *}$ & $0.021^{* *}$ & $0.003^{* *}$ & $0.058^{\mathrm{ns}}$ & $72.25^{\mathrm{ns}}$ \\
\hline \multirow[t]{2}{*}{$\begin{array}{l}\text { Year } \times \text { Treat } \\
\text { ment }\end{array}$} & - & 9 & $0.016^{\mathrm{ns}}$ & $0.003^{\mathrm{ns}}$ & $3.546^{* *}$ & $0.039^{\mathrm{ns}}$ & $0.002^{\mathrm{ns}}$ & ${ }_{\mathrm{s}}^{0.0003^{\mathrm{n}}}$ & $0.007^{\mathrm{ns}}$ & $8.027^{\mathrm{ns}}$ \\
\hline & 2011 & 18 & 0.034 & 0.004 & 0.079 & 0.007 & 0.004 & 0.001 & 0.008 & 13.32 \\
\hline \multirow[t]{2}{*}{ Error } & 2012 & 18 & 0.035 & 0.027 & 0.386 & 0.036 & 0.003 & 0.001 & 0.101 & 0.723 \\
\hline & Means & 38 & 0.052 & 0.016 & 0.224 & 0.021 & 0.004 & 0.001 & 0.052 & 6.66 \\
\hline \multirow[t]{3}{*}{$\mathrm{CV}(\%)$} & 2011 & & 11.52 & 7.02 & 19.38 & 4.00 & 14.75 & 12.64 & 4.49 & 24.20 \\
\hline & 2012 & & 9.81 & 8.25 & 17.06 & 8.07 & 10.95 & 9.30 & 9.96 & 4.92 \\
\hline & Means & & 13.03 & 8.72 & 18.63 & 6.51 & 13.74 & 10.54 & 8.80 & 15.94 \\
\hline
\end{tabular}

In each column, $* *, *$, and ${ }^{\text {ns }}$ means significant at $0.01,0.05$ probability level, and non-significant, respectively.

In relation to the mean comparisons in the first growth season (2011), the highest value of capitula dry weight, leaves dry weight, total dry weight, total carbohydrates of capitula, and total flavonoids of leaves was obtained in treatment of 1.5 L.ha ${ }^{-1}$ humiforte $\left(\mathrm{H}_{2}\right)$. The Maximum amount of total carbohydrates, phosphorous and potassium of leaves was related to treatment of $1.5 \mathrm{~L} \cdot \mathrm{ha}^{-1}$ kadostim $\left(\mathrm{K}_{2}\right)$. The most content of nitrogen, $\mathrm{Cu}$, and $\mathrm{Mn}$ was observed in treatment of $1.5 \mathrm{~L}^{\mathrm{ha}}{ }^{-1}$ fosnutren $\left(F_{2}\right)$. The highest amount of total flavonoids of capitula, and $\mathrm{Zn}$ was recorded in treatment of $1.5 \mathrm{~L} . h a^{-1}$ aminolforte $\left(\mathrm{A}_{2}\right)$. The maximum content of $\mathrm{Fe}$ and $\mathrm{Ca}$ was produced in treatment of $75 \mathrm{~kg} \cdot \mathrm{ha}^{-1}$ chemical complete fertilizer (CF) (Table 3). On the other hand, the lowest amount of leaves and capitula dry weight, total dry weight, content of $\mathrm{N}, \mathrm{P}, \mathrm{K}$, and $\mathrm{Zn}$ in treatment of control $(\mathrm{C})$, total carbohydrates of capitula and leaves in 1.5 L.ha ${ }^{-1}$ aminolforte $\left(\mathrm{A}_{2}\right)$, total flavonoids of capitula and $\mathrm{Cu}$ in treated plants with $0.75 \mathrm{~L} \cdot \mathrm{ha}^{-1}$ humiforte $\left(\mathrm{H}_{1}\right)$, total flavonoids of leaves and content of $\mathrm{Fe}, \mathrm{Mn}$ and $\mathrm{Ca}$ in treated plants with 0.75 L.ha ${ }^{-1}$ kadostim $\left(\mathrm{K}_{1}\right)$ was observed (Table 3). With consideration of mean comparisons in the second growth season (2012), the highest amount of total dry weight, total flavonoids of leaves, and content of potassium was attained by $1.5 \mathrm{~L}^{\mathrm{L}} \mathrm{ha}^{-1}$ humiforte $\left(\mathrm{H}_{2}\right)$, while the most content of iron and calcium was observed in treatment of 75 kg.ha ${ }^{-1}$ chemical complete fertilizer (Table 3). The lowest total dry weight and content of potassium in treatment of control (C), total flavonoids of leaves in $1.5 \mathrm{~L}^{-\mathrm{ha}^{-1}}$ aminolforte $\left(\mathrm{A}_{2}\right)$ was obtained. Also, content of iron and calcium at 1.5 and 0.75 L.ha $^{-1}$ kadostim was reduced to the lowest amount, respectively (Table 3).

Table 3. Mean comparisons effects of bio-stimulators on the measured parameters of pot marigold (Calendula officinalis L) during 2011 and 2012 growth season

\begin{tabular}{|c|c|c|c|c|c|c|c|c|c|c|c|c|c|c|}
\hline \multirow{3}{*}{$\begin{array}{l}\text { Treat- } \\
\text { ments }\end{array}$} & \multirow{2}{*}{\multicolumn{2}{|c|}{$\begin{array}{l}\text { Capitula dry } \\
\text { weight }\left(\mathrm{g} \cdot \mathrm{m}^{-2}\right)\end{array}$}} & \multirow{2}{*}{\multicolumn{2}{|c|}{$\begin{array}{l}\text { Leaves dry } \\
\text { weight }\left(\mathrm{g} \cdot \mathrm{m}^{-2}\right)\end{array}$}} & \multirow{2}{*}{\multicolumn{2}{|c|}{ Total dry weight $\left(\mathrm{g} \cdot \mathrm{m}^{-2}\right)$}} & \multicolumn{4}{|c|}{ Total carbohydrates (mg.g $\left.{ }^{-1} \mathrm{DW}\right)$} & \multicolumn{4}{|c|}{ Total flavonoids (mg.g $\left.{ }^{-1} \mathrm{DW}\right)$} \\
\hline & & & & & & & \multicolumn{2}{|c|}{ Capitula } & \multicolumn{2}{|l|}{ Leaves } & \multicolumn{2}{|c|}{ Capitula } & \multicolumn{2}{|c|}{ Leaves } \\
\hline & 2011 & 2012 & 2011 & 2012 & 2011 & 2012 & 2011 & 2012 & 2011 & $\begin{array}{l}201 \\
2\end{array}$ & 2011 & 2012 & 2011 & 2012 \\
\hline $\mathrm{C}$ & $26.73^{\mathrm{d}}$ & 35.31 & $17.91^{\mathrm{d}}$ & 21.92 & $100.89^{\mathrm{d}}$ & $86.50^{\mathrm{c}}$ & $0.23^{\mathrm{bc}}$ & 0.43 & $0.18^{\mathrm{bcd}}$ & 0.22 & $0.17^{\mathrm{cd}}$ & 0.49 & $0.07^{\mathrm{bc}}$ & $0.11^{\mathrm{abc}}$ \\
\hline $\mathrm{CF}$ & $42.33^{\mathrm{ab}}$ & 43.11 & $22.89^{\mathrm{abc}}$ & 20.41 & $109.44^{\text {cd }}$ & $135.61^{b}$ & $0.22^{\mathrm{cd}}$ & 0.44 & $0.19^{\mathrm{ab}}$ & 0.23 & $0.23^{\mathrm{ab}}$ & 0.52 & $0.09^{\mathrm{ab}}$ & $0.11^{\mathrm{abc}}$ \\
\hline $\mathrm{A}_{1}$ & $29.46^{\text {bcd }}$ & 36.75 & $20.20^{\mathrm{cd}}$ & 23.03 & $131.13^{\mathrm{abcd}}$ & $150.72^{\mathrm{ab}}$ & $0.23^{\mathrm{bc}}$ & 0.44 & $0.15^{\mathrm{de}}$ & 0.21 & $0.23^{\mathrm{ab}}$ & 0.52 & $0.07^{\mathrm{bc}}$ & $0.10^{\mathrm{bc}}$ \\
\hline $\mathrm{A}_{2}$ & $38.06^{\mathrm{abcd}}$ & 40.34 & $21.40^{\mathrm{bc}}$ & 24.96 & $150.42^{\mathrm{ab}}$ & $160.40^{\mathrm{ab}}$ & $0.21^{\mathrm{d}}$ & 0.42 & $0.14^{\mathrm{e}}$ & 0.20 & $0.25^{\mathrm{a}}$ & 0.53 & $0.09^{\mathrm{ab}}$ & $0.08^{\mathrm{d}}$ \\
\hline $\mathrm{K}_{1}$ & $38.33^{\mathrm{abcd}}$ & 41.11 & $21.86^{\mathrm{bc}}$ & 22.91 & $124.95^{\mathrm{bcd}}$ & $147.62^{\mathrm{ab}}$ & $0.22^{\mathrm{cd}}$ & 0.43 & $0.18^{\mathrm{abc}}$ & 0.22 & $0.15^{\mathrm{cd}}$ & 0.48 & $0.04^{\mathrm{d}}$ & $0.09^{\mathrm{d}}$ \\
\hline $\mathrm{K}_{2}$ & $42.53^{\mathrm{ab}}$ & 43.21 & $23.57^{\mathrm{ab}}$ & 22.72 & $140.62^{\mathrm{abc}}$ & $155.50^{\mathrm{ab}}$ & $0.24^{\mathrm{bc}}$ & 0.41 & $0.21^{\mathrm{a}}$ & 0.23 & $0.23^{\mathrm{ab}}$ & 0.52 & $0.05^{\mathrm{cd}}$ & $0.09^{\mathrm{cd}}$ \\
\hline $\mathrm{F}_{1}$ & $27.93^{\mathrm{cd}}$ & 35.91 & $20.89^{\text {bcd }}$ & 23.41 & $118.77^{\mathrm{bcd}}$ & $144.52^{\mathrm{ab}}$ & $0.25^{\mathrm{b}}$ & 0.45 & $0.17^{\text {bcd }}$ & 0.21 & $0.19^{b c}$ & 0.50 & $0.06^{\mathrm{cd}}$ & $0.10^{\mathrm{cd}}$ \\
\hline $\mathrm{F}_{2}$ & $33.33^{\mathrm{abcd}}$ & 38.61 & $23.89^{\mathrm{ab}}$ & 24.91 & $117.57^{\mathrm{bcd}}$ & $143.92^{\mathrm{ab}}$ & $0.25^{\mathrm{b}}$ & 0.44 & $0.16^{\text {cde }}$ & 0.21 & $0.23^{\mathrm{ab}}$ & 0.52 & $0.08^{\mathrm{b}}$ & $0.11^{\mathrm{abc}}$ \\
\hline $\mathrm{H}_{1}$ & $41.00^{\mathrm{abc}}$ & 42.43 & $22.0^{\mathrm{bc}}$ & 23.93 & $148.80^{\mathrm{ab}}$ & $159.53^{\mathrm{ab}}$ & $0.26^{\mathrm{ab}}$ & 0.45 & $0.17^{\text {bcde }}$ & 0.21 & $0.12^{\mathrm{d}}$ & 0.46 & $0.09^{\mathrm{ab}}$ & $0.116^{\mathrm{ab}}$ \\
\hline $\mathrm{H}_{2}$ & $44.00^{\mathrm{a}}$ & 43.93 & $25.24^{\mathrm{a}}$ & 24.24 & $161.26^{\mathrm{a}}$ & $165.81^{\mathrm{a}}$ & $0.28^{\mathrm{a}}$ & 0.46 & $0.19^{\mathrm{ab}}$ & 0.22 & $0.22^{\mathrm{ab}}$ & 0.52 & $0.10^{\mathrm{a}}$ & $0.12^{\mathrm{a}}$ \\
\hline
\end{tabular}

* Means in each column followed by the same letter are not significantly different $(P<0.01)$. 
RAFIEE H., et al.

Table 3. continued

\begin{tabular}{|c|c|c|c|c|c|c|c|c|c|c|c|c|c|c|c|c|}
\hline \multirow[t]{2}{*}{$\begin{array}{l}\text { Treat- } \\
\text { ments }\end{array}$} & \multicolumn{2}{|c|}{$\mathrm{N}(\%)$} & \multicolumn{2}{|c|}{$\begin{array}{c}\mathrm{P} \\
\left(\mathrm{mg} \cdot \mathrm{g}^{-1}\right. \\
\mathrm{DW})\end{array}$} & \multicolumn{2}{|c|}{$\begin{array}{c}\mathrm{K} \\
\left(\mathrm{mg} \cdot \mathrm{g}^{-1}\right. \\
\mathrm{DW})\end{array}$} & \multicolumn{2}{|c|}{$\begin{array}{c}\mathrm{Fe} \\
\left(\mathrm{mg} \cdot \mathrm{g}^{-1}\right. \\
\mathrm{DW})\end{array}$} & \multicolumn{2}{|c|}{$\begin{array}{c}\mathrm{Zn} \\
\left(\mathrm{mg} \cdot \mathrm{g}^{-1}\right. \\
\mathrm{DW})\end{array}$} & \multicolumn{2}{|c|}{$\begin{array}{c}\mathrm{Cu} \\
\left(\mathrm{mg} . \mathrm{g}^{-1}\right. \\
\mathrm{DW})\end{array}$} & \multicolumn{2}{|c|}{$\begin{array}{c}\mathrm{Mn} \\
\left(\mathrm{mg} \cdot \mathrm{g}^{-1}\right. \\
\mathrm{DW})\end{array}$} & \multicolumn{2}{|c|}{$\begin{array}{c}\mathrm{Ca} \\
\left(\mathrm{mg} \cdot \mathrm{g}^{-1}\right. \\
\mathrm{DW})\end{array}$} \\
\hline & 2011 & 2012 & 2011 & 2012 & 2011 & 2012 & 2011 & 2012 & 2011 & 2012 & 2011 & 2012 & 2011 & 2012 & 2011 & 2012 \\
\hline $\mathrm{C}$ & $1.34^{\mathrm{d}}$ & 1.77 & $0.75^{\mathrm{d}}$ & 1.92 & $0.81^{\mathrm{e}}$ & $1.39^{\mathrm{h}}$ & $1.95^{\mathrm{bcd}}$ & $2.32^{b}$ & $0.34^{\mathrm{c}}$ & 0.46 & $0.23^{\mathrm{cd}}$ & 0.33 & $1.88^{\mathrm{cd}}$ & 3.14 & $18^{\mathrm{ab}}$ & $18.74^{\mathrm{b}}$ \\
\hline $\mathrm{CF}$ & $1.65^{\mathrm{abcd}}$ & 1.93 & $0.93^{\mathrm{abc}}$ & 2.01 & $1.32^{\text {cde }}$ & $1.89^{\mathrm{fgh}}$ & $2.80^{\mathrm{a}}$ & $2.74^{\mathrm{a}}$ & $0.44^{\text {bc }}$ & 0.51 & $0.24^{\mathrm{bcd}}$ & 0.33 & $1.98^{\mathrm{bc}}$ & 3.19 & $24.50^{\mathrm{a}}$ & $21.99^{\mathrm{a}}$ \\
\hline $\mathrm{A}_{1}$ & $1.50^{\mathrm{cd}}$ & 1.85 & $0.90^{\mathrm{bc}}$ & 2.00 & $1.52^{\mathrm{bcd}}$ & $2.39^{\mathrm{bcd}}$ & $1.90^{\mathrm{cd}}$ & $2.29^{\mathrm{b}}$ & $0.37^{\mathrm{c}}$ & 0.47 & $0.22^{\mathrm{cd}}$ & 0.33 & $1.88^{\mathrm{cd}}$ & 3.14 & $10.50^{c}$ & $14.99^{\mathrm{cd}}$ \\
\hline $\mathrm{A}_{2}$ & $1.58^{\mathrm{bcd}}$ & 1.89 & $0.83^{\mathrm{cd}}$ & 1.97 & $1.93^{\mathrm{ab}}$ & $2.89^{\mathrm{efg}}$ & $2.04^{\mathrm{bcd}}$ & $2.36^{\mathrm{b}}$ & $0.58^{\mathrm{a}}$ & 0.58 & $0.28^{\mathrm{ab}}$ & 0.35 & $2.00^{\mathrm{bc}}$ & 3.20 & $18.00^{\mathrm{ab}}$ & $18.74^{\mathrm{b}}$ \\
\hline $\mathrm{K}_{1}$ & $1.61^{\mathrm{bcd}}$ & 1.91 & $0.80^{\mathrm{cd}}$ & 1.95 & $1.37^{\mathrm{cd}}$ & $3.39^{\mathrm{def}}$ & $1.89^{\mathrm{d}}$ & $2.29^{\mathrm{b}}$ & $0.38^{\mathrm{bc}}$ & 0.48 & $0.26^{\mathrm{abc}}$ & 0.35 & $1.78^{\mathrm{d}}$ & 3.09 & $10.00^{\mathrm{c}}$ & $14.74^{\mathrm{d}}$ \\
\hline $\mathrm{K}_{2}$ & $1.72^{\mathrm{abc}}$ & 1.96 & $1.04^{\mathrm{a}}$ & 2.07 & $2.13^{\mathrm{a}}$ & $3.88^{\text {cde }}$ & $2.05^{\mathrm{bc}}$ & $2.04^{\mathrm{b}}$ & $0.50^{\mathrm{ab}}$ & 0.53 & $0.24^{\mathrm{bcd}}$ & 0.34 & $1.94^{\mathrm{cd}}$ & 3.17 & $10.30^{\mathrm{c}}$ & $15.01^{\mathrm{d}}$ \\
\hline $\mathrm{F}_{1}$ & $1.39^{\mathrm{cd}}$ & 1.80 & $0.93^{\mathrm{abc}}$ & 2.01 & $1.32^{\text {cde }}$ & $4.39^{\mathrm{bcd}}$ & $2.09^{b}$ & $2.39^{\mathrm{b}}$ & $0.40^{\mathrm{bc}}$ & 0.49 & $0.28^{\mathrm{ab}}$ & 0.36 & $1.98^{\mathrm{bc}}$ & 3.18 & $12.84^{\mathrm{bc}}$ & $16.16^{\mathrm{cd}}$ \\
\hline $\mathrm{F}_{2}$ & $1.98^{\mathrm{a}}$ & 2.09 & $0.98^{\mathrm{ab}}$ & 2.04 & $1.72^{\mathrm{abc}}$ & $4.89^{\mathrm{abc}}$ & $2.11^{\mathrm{b}}$ & $2.40^{\mathrm{b}}$ & $0.35^{\mathrm{c}}$ & 0.47 & $0.31^{\mathrm{a}}$ & 0.37 & $2.20^{\mathrm{a}}$ & 3.30 & $13.33^{b c}$ & $16.40^{\mathrm{c}}$ \\
\hline $\mathrm{H}_{1}$ & $1.43^{\mathrm{cd}}$ & 1.81 & $1.00^{\mathrm{ab}}$ & 2.05 & $1.22^{\text {cde }}$ & $5.42^{\mathrm{ab}}$ & $2.02^{\mathrm{bcd}}$ & $2.35^{\mathrm{b}}$ & $0.44^{\text {bc }}$ & 0.51 & $0.21^{\mathrm{d}}$ & 0.32 & $2.12^{\mathrm{ab}}$ & 3.26 & $17.00^{\mathrm{bc}}$ & $18.24^{\mathrm{b}}$ \\
\hline $\mathrm{H}_{2}$ & $1.86^{\mathrm{ab}}$ & 2.03 & $0.80^{\mathrm{cd}}$ & 1.95 & $1.11^{\mathrm{de}}$ & $5.9^{\mathrm{a}}$ & $2.04^{\mathrm{bcd}}$ & $2.36^{\mathrm{b}}$ & $0.50^{\mathrm{ab}}$ & 0.54 & $0.24^{\text {bcd }}$ & 0.34 & $2.14^{\mathrm{ab}}$ & 3.27 & $16.67^{\mathrm{bc}}$ & $18.08^{\mathrm{b}}$ \\
\hline
\end{tabular}

* Means in each column followed by the same letter are not significantly different $(P<0.01)$.

\section{DISCUSSION}

Foliar application of bio-stimulators was appeared to have significant and positive effect on morphological and phytochemical parameters and elements content of pot marigold. Treated plants at $1.5 \mathrm{~L} \mathrm{ha}^{-1}$ humiforte showed increase in yield of capitula dry weight to $39.25 \%$ in 2011 . The results of this study are in agreement with the experiment results of Nahed et al. (2009a) on Gladiolus grandflorum L. and Nahed et al. (2009b) on Antirrhinum majus L. (14, 15). Neeraja et al. (2005) reported that application of amino acids increased flowers number, fruit set and yield of fruits (23). Thon et al. (1981) showed that amino acids supply sources of nitrogen for plant cells that can be absorbed more quickly than inorganic nitrogen by cells (16). In year of 2011, treatment of 1.5 L.ha $^{-1}$ humiforte caused increase in yield of leaves dry weight to $29.04 \%$. According to the results of Celik and katkat (2007) experiment, application of macro elements (existed in humiforte formulation) had positive effect on leaves and roots dry weight of Zea mays (17). Poly amines have fundamental role in control of cell cycle, cell division, morphogenesis in phytochrome, plant hormone and plant senescence in response to environmental stresses and they increase yield $(18,19)$. Amino acids are the precursor of polyamines that are essential in the regulation of plant growth and development (47, 48). Polyamines also act as signal molecules to regulate gene activity related to cellular $\mathrm{N}$ metabolism and the metabolism of several amino acids such as proline, arginine, $\gamma$ aminobutyric acid (GABA), ornithine, and glutamic acid, all of which play important roles in plant responses to higher $\mathrm{N}$ exposure $(49,50$, 51). In consideration of mean comparisons in two years, the most total dry weight was observed in plants treated by 1.5 L.ha ${ }^{-1}$ humiforte with yield increase to 37.43 and $18.21 \%$ in comparison with control in 2011 and 2012, respectively. In spite of better climate condition in second year, the increasing amount of yield in first year was more than second year affected by bio-stimulators application. It showed that amino acids compounds caused more vegetative growth in environmental stresses. These results are according to the results of Haj Seyed Hadi et al. (2011) on Matricaria recutita L., Khalid et al. (2006) on Calendula officinalis L. and Ezz El-din et al. (2010) on Carum carvi L. (20, 21, 22). It is recently reported that application of amino acids increased growth and yield of tomato cultivars $(24,25)$. Maybe the positive effect of amino acids is because of the intercellular function as an osmotic adapter (26). Because they are so soluble in water and they increase concentration of osmotic compounds in cells. Treated plants with amino acid compounds showed an increase in carbohydrates and flavonoids content of leaves and capitula compared to control treatment in 2011 in contrast to 2012. The reason for that can be the more environmental stresses in 2011 and this leads to more synthesis of secondary metabolites like flavonoids (52, 53). According to the results of Refaat and Naghib (1998), application of amino acids on Capsicum annum L. leaves, increased carbohydrates in these plants. Maybe increasing effect of amino acids is related to their effect on the biosynthesis of chlorophyll molecules that influences the content of total carbohydrates. It is reported that succinyl-CoA (one of the metabolites of Krebs cycle) and amino acid of glycine stimulate the biosynthesis pathway of 
chlorophyll $(27,28)$. The obtained results are in line with the results of Abou Dahab et al. (2006) on Philodendron plant (29). Treatment of arginine on Vigna radiata L. increased content of soluble sugar, poly saccharids, total carbohydrates, proline, total amino acids and protein content of vigna seeds in irrigation with saline and no saline water (30).

In first year, the most content of nitrogen was obtained in foliar application of $1.5 \mathrm{~L}^{\mathrm{h} \mathrm{ha}^{-1}}$ fosnutren. As results of Abdul Qados (2010), application of amino acids on seeds and leaves of Vigna radiata L. increased content of nitrogen and dry weight of leaves (30). Amino acids possibly increase activities of metabolic pathways in plants and plants yield will improve by increasing efficiency of roots in absorption of macronutrients from soil (56). Fosnutren treatment has two elements of nitrogen and phosphorous in its compound that maybe the reason for high content of nitrogen is synergistic effect of these two elements on each other (31) and so existence of lowest amount of phosphorous can increase amount of nitrogen in leaves. Phosphorous content in leaves reached the highest amount in plants treated by $1.5 \mathrm{~L}^{-h^{-1}}$ kadostim in 2011. Our results are similar to results of Abdul-Qados (2010) with application of arginine amino acid on Vigna radiata $\mathrm{L}$. and El-Ghamry et al. 2009 with application of amino acids and humic acid on Vicia faba L. (30, 32). The amino acids, used in formulation of biostimulators, can improve qualitative and quantitative traits with increase of mRNA transcription to 2.5 fold, activation of effective hormones in reproductive growth, activation of carbohydrates synthesis, increase of absorption and translocation of elements and increase of protein content in plants in environmental stresses $(33,5,34)$. Plants treated by $1.5 \mathrm{~L}^{\mathrm{h}} \mathrm{ha}^{-1}$ kadostim and humiforte showed the most content of potassium in two growth seasons of 2011 and 2012, respectively. These results are in agreement with research data of Jill et al. (2011) on three species of Ericaceae (Vaccinium myrtilloides, Ledum groenlandicum, and Chamaedaphne calyculata) (35). Humiforte and kadostim have macro element of potassium in their formulation and this is the reason for high amount of this element in leaves in two years. In deed the result for higher amount of this element in second year may be the climate conditions, as in second year, lower temperature and higher amount of rainfall forces the plant to conserve higher content of potassium in its vegetative parts, while in first year effect of amino acid compounds was more significant in lower amount of potassium because of more stresses. Potassium is known as one of important nutritional elements that effects yield and quality of grains and fruits. This nutritional element plays a fundamental role in growth and metabolism (36). Also it activates enzymes and acts as an osmotic adaptor for keeping turgid pressure. This element adopts opening and closing of stomata and anion charges $(37,38)$. Fe content increased to the highest amount with application of chemical fertilizer in comparison with control and other treatments while it reached the lowest amount in treatment of kadostim in both years. These results are similar to results of Celik et al. (2010) on Zea mays, Mottaghian et al. (2008) on soybean cultivars and saha et al. (2005) on Aloe vera plants (39, $52,53)$. Fe content in leaves of soybean cultivars by application of municipal compost enriched with chemical fertilizer and in Aloe vera plants by chemical fertilizers was the highest $(54,55)$. Application of excessive amount of potassium (main element in kadostim) causes inhibition of iron absorption and may improve chlorosis of iron (39). Urrestarazu et al. (1994) reported that plants absorb potassium more than iron and excessive amounts of potassium inhibits absorption and translocation of iron in plants and it leads to lack of iron (40). Recent studies showed that while symptoms of chlorosis are appeared high amount of potassium will be found in chlorotic plant samples $(41,17)$. In deed maybe application of $75 \mathrm{~kg} \cdot \mathrm{ha}^{-1}$ chemical complete fertilizer in soil decreases $\mathrm{pH}$ in rhizosphere and it causes more uptake of $\mathrm{Fe}$ element in plant (46). Foliar application of aminolforte with concentration of $1.5 \mathrm{~L}^{\mathrm{h}} \mathrm{ha}^{-1}$ caused the highest amount of $\mathrm{Zn}$ element in leaves in 2011. Similar results have been reported by Abdel-Mawgoud et al. (2011) on Phasaeolous vulgaris L. in a way that foliar application of amino acids and maniplex increased content of elements $\mathrm{Mn}, \mathrm{Fe}$ and $\mathrm{Zn}$ (42). Amino acids with chelating effect on micronutrients cause easier absorption and transportation of them inside the plant system. This effect is due to the effect on cell membrane permeability (57). Results of this experiment are agreeable with those of Fawzy et al. (2011) on Allium sativum L. (43). Application of amino acids in experimental year of 2011 increased $\mathrm{Cu}$ element content in comparison with control treatment. Result of increase in content of $\mathrm{Cu}$ at treatment of $1.5 \mathrm{~L}^{-h^{-1}}$ fosnutren is related to 
existence of macro element of $\mathrm{N}$ in this formulation and synergistic effect between $\mathrm{N}$ and $\mathrm{Cu}$. In spite of existence of phospurous in this formulation that has antagonistic effect with $\mathrm{Cu}$, due to higher content of $\mathrm{N}$ and more effective amino acids applied at 1.5 L.ha $^{-1}$ fosnutren the synergistic effect is dominant (31). Element of $\mathrm{Mn}$ increased with application of fosnutren treatments in first year. These results are as the same of Abdel-Mawgoud et al. (2011) results on Phasaeolous vulgaris L., Fawzy et al. (2012) on Allium sativum L. and Yousef et al. (2011) on olive seedlings (42, 43, 44). Amino acid compounds can improve fertilizer assimilation, increase uptake of nutrients and water, enhance the photosynthetic rate and dry matter partitioning, and hence increase crop yield (58). Maybe because of synergistic effect between $\mathrm{N}$ (an element in this formulation) and micro nutrient of $\mathrm{Mn}$, this element reached the highest amount in treatment of $1.5 \mathrm{~L} \cdot \mathrm{ha}^{-1}$ fosnutren (31). The result for effect of chemical fertilizer in high amount of $\mathrm{Ca}$ in leaves in both years is according to results of Abdul-Qados (2010) on Vigna radiata L. and Abdel-Mawgoud (2011) $(30,42)$. The lowest amount of this element was reached in 0.75 L.ha ${ }^{-1}$ kadostim in both years and this result can be because of antagonistic effect of potassium (an element in kadostim formulation) and calcium on each other in this compound (31).

\section{CONCLUSION}

According to these results, bio-stimulators with formulation of amino acids caused improve in the morphological and phytochemical traits and amount of mineral elements in Calendula officinalis L. as a medicinal plants. Also, the bio-stimulators includings humiforte, kadostim and aminolforte could be recommended to improve growth and phytochemical parameters of $C$. officinalis in environmental stress conditions. These results can be due to existence of amino acids and macro elements of $\mathrm{N}, \mathrm{P}$ and $\mathrm{K}$ in these compounds.

\section{ACKNOWLEDGMENTS}

This research was funded by the Cultivation and Development Department of Medicinal Plants Research Center at the Institute of Medicinal Plants (IMP) affiliated with the Academic Center for Education Culture and Research (ACECR) in Karaj, Iran.

\section{REFERENCES}

1. Khan, M.U., Ankur, R., Deepika, B., Shadan, A., Seema, R., and Ansari, S.H. Diverse belongings of Calendula officinalis: An
RAFIEE H., et al.

overview. International Journal of Pharmaceutical Sciences and Drug Research;3(3): 173-177. 2011.

2. Lea, A.G.H., HPLC in food analysis. Academic Press, London, p. 277. 1988.

3. Meda, A., Laien, C.E., Romito, M., Millogo, J., Nacoulma, G.O., Determination of the total phenolic, flavonoid and proline contents in Burkina Fasan honey, as well as their radical scavenging activity. Food Chem. 2005. 91, 571-577.

4. Starck, Z., Stosowanie regulatorów wzrostu oraz biostymulatorów $\mathrm{w}$ uprawie roœlin. Rolnik Dzierawca. Luty.74-76. 2005.

5. El-Kady, A.F., Growth and yield of sunflower as influenced by some micronutrients. Adv. Res. 1997. 2: 43.

6. Naghuib, N.Y., Khalil, M.Y., and El Sherbeny, S.E., A comparative Study on the productivity and chemical constittents of various sources and species of Calendula plants as affected by two foliar fertilizers. Journal of Applied Sciences Research. 1(2): 176-189, 2005.

7. Mandal, A.K.A., Kumar, R.R., and Thomas, J., An overview of PGR trials in UPASI TRF. Planters Chron., 103:12-16. 2007.

8. Quettier, D.C., Gressier, B., Vasseur, J., Dine, T., Brunet, C., Luyckx, M.C., Cayin, J.C., Bailleul, F., and Trotin, F., Phenolic compounds and antioxidant activities of buckwheat (Fagopyrum esculentum Moench) hulls and flour. J. Ethnopharmacol. 2000. 72: $35-42$.

9. Stankovic, M.S., Total phenolic content, flavonoid concentration and Antioxidant Activity of Marrubium peregrinum L. Extracts. 2011.

10.Dubois, M., Smith, F., Gilles, K.A.G., Hamilton, J.K., and Robers, P.A., Colorimetric method for determination of sugars and related substances. Anal. Chem., 28: 350-356. 1966.

11.Wahing, I., Van, W., Houba, V.J.G., and Van der lee, J.J., Soil and plant analysis, a series of syllabi. part7, plant analysis procedure. Wageningen Agriculture University. 1989.

12.Chapman, H.D., and Pratt, P.F., Method of analysis for soils, plants and waters. University of California. Division of agricultureal Sciences. 1961.

13.García, R., Belmont, R., Padilla, H., Torres, M.C. and Báez, A., "Trace metals and inorganic ions measurements in rain from Mexico City and a nearby rural area". Rev. Chemistry and Ecology. 25(2), pp. 71-86. 2009. 
14.Nahed, G.A.A, Lobna, S.T., and Ibrahim, S.M.M., Some studies on the effect of Putrescine, Ascorbic Acid and Thiamine on growth, flowering and chemical Constituents of Gladiolus plants at Nubria. Ozean Journal of Applied Sciences 2(2).p:169-179. 2009a.

15.Nahed, G.A.A, Mona, H.M., and Azza, A.M.M., Physiological effect of Phenylalanine and Tryptophan on the growth and chemical constitutes of Antirrihinum Majus plants.Ozean Journal of Applied Sciences. 2(4). 399- 407. 2009b.

16.Thon, M., Maretzki, A., Korner, E., and Sokai. W.S., Nutrient uptake and accumulation by sugar cane cell culture in relation to growth cycle. Plant cell Tissue and Organ Culture, (1): 3-14. 1981.

17.Celik, H., and Katkat, A.V., Some physical soil properties and potassium as an intensified factor on iron chlorosis. International Journal of Soil Science. vol. 2, No. 4, p.294-300. 2007.

18. Walters, DR., Polyamine in plant-microbe interactions. Physiol Mol Plant Pathol57: 137-146. 2000.

19.Abd El-Monem, A.A., Polyamines as modulators of wheat growth, metabolism and reproductive development under temperature stress. PhD Thesis; Ain Shamas University (Cairo, Egypt). 2007.

20.Haj Seyed Hadi, M.R., Darz, M.T., Ghandehari, Z. and Riazi., G., Effects of Vermicompost and amino acids on the flower yield and essential oil production from Matricaria chamomile L. Journal of Medicinal Plants Research. Vol. 5(23), pp. 5611-5617. 2011.

21.Khalid, Kh.A., Yassen, A.A. and Zaghloul. M., Effect of Soil Solarization and Cattle Manure on the growth, essential oil and chemical composition of Calendula officinalis L. plants. Journal of Applied Sciences Research 2(3): 142-152. 2006.

22.Ezz El-Din, A.A., Hendawy, S.F., Aziz, E.E. and Omer, E.A., Enhancing growth, yield and essential oil of Caraway plants by nitrogen and potassium Fertilizers. International Journal of Academic Research. Vol. 2(3) 192- 197. 2010.

23.Neeraja, G., Reddy, I.P., and Gautham, B., Effect of growth promoters on growth and yield of tomato cv. Marutham. Journal of Research ANGRAU. 33(3): 68-75. 2005.

24.Hafez, M.R., Impact of some chemical treatments on salinity tolerance of some tomato cultivars. M.Sc. thesis, Fac. Agric., Ain Shams University, pp. 93.2001.

25.Abdel-Latif, A.M., Physiological studies on tomato. M.Sc. thesis. Fac. Agric., Cairo University, Egypt. 1995.

26. Treichel, S., The effect of $\mathrm{NaCl}$ on the concentration of proline in different halophytes. Z.Pflanzen physiol. 76: 5668. (c.f. Amer. J. Bot. 66 (3): 307- 312). 1975.

27.Refaat, A.M. and Naguib, N.Y., Peppermint yield and oil quality as affected by application of some amino acids. Bull. Fac. Agric. Univ. Cairo, 49: 89-98. 1998.

28.Devlin, R.M., Plant physiology 2nd Ed. P.164, S. Muthiah at Tamil and Printers and Traders Pvt. Ltd., Madra, India. 1969.

29.Abou Dahab, T.A.M., and Nahed, G.A.A., Physiological effect of Diphenylamin and Tryptophan on the growth and chemical constituents of Philodendron erubescens plants. World Journal of Agricultural Sciences. 2(1):75-81. 2006.

30.Abdul Qados, A.M.S., Effect of arginine on growth, nutrient composition, yield and nutritional value of mung bean plants grown under salinity stress. Nature and Science. 8(7). 30-42. 2010.

31.Nijar, J.S., Nutrition of fruit trees. Translation: Ardalan, M.M., Gh. R. Savaghebi Firoozabadi. Publications of Jahad Daneshgahi Tehran. pp: 260. 2009.

32.Ayman, M.G., Kamar, M.A.H. and Khalid, M.G., Amino and humic acids promote growth, yield and disease Resistance of Faba Bean cultivated in clay soil. Australian Journal of Basic and Applied Sciences, 3(2):731-739. 2009.

33. Research and development unit of Inagropars. Inagropars production (AgroBiological industries Co.). No. 21, Golestan II, Pasdaran Ave. Tehran 16669 Iran. 16 pp. 2010.

http://www.inagropars.com/Catalogue.pdf.

34. Thomas, J., Mandal, AKA., Raj Kumar, R., and Chordia, A., Role of biologically active amino acid formulations on quality and crop productivity of Tea (Camellia sp.). Int. J. Agric. Res.; 4: 228 - 236. 2009.

35.Jill, L.B., Rose, S., Sari, J., Tim, R.M., Rakesh, M., Stephanie, L. and Subhash M., Effects of nutrient on leaf addition on leaf chemistry, morphology, and photosynthetic capacity of three bog shrubs. Oecologia. 167:355-368. 2011.

36.Ruiz, J.M., and Romero, L., Relationship between potassium fertilization and nitrate 
assimilation in leaves and fruits of cucumber (Cucumis sativus L.) plants. Annals of Applied Biology. vol. 140, p.241-245. 2002.

37.Marschner, H., Mineral Nutrition of higher plants. Academic Press, London p. 912. 1995.

38.Mengel, K., Potassium: handbook of plant nutrition-Boca Raton, USA, p.91-120. 2007.

39.Celik, H., Asik, B.B., Gurel, S. and Katkat, A.V., Effects of potassium and iron on macro element uptake of maize. ZemdirbysteAgriculture. 97(1):11-22. 2010.

40.Urrestarazu, M., Sanchez, A. and Alvarado J., Iron indices and micronutrients in deciduous fruit trees. Communications in Soil Science and Plant Analysis. vol. 25, No. 910, p. 1685-1751. 1994.

41.Torres, R.M., Barra, J.D.E., and Conzales, G.A., Morphological changes in leaves of Mexican lime affected by iron chlorosis. Journal of Plant Nutrition. vol. 29, No. 4, p. 615-628. 2006.

42.Abdel-Mawgoud, A.M.R., El-Bassiouny, A.M., Ghoname, A. and Abou-Hussein, S.D., Foliar application of amino acids and micronutrients enhance performance of Green Bean crop under newly reclaimed land conditions. Australian Journal of Basic and Applied Sciences. 5(6): 51-55. 2011.

43.Fawzy, Z.F., El-shal, Z.S., Yunsheng, L., Quyang, Z. and Omaima, M.S., Response of Garlic (Allium Sativum L.) plants to foliar spraying stimulants under sandy soil condition. Journal of Applied Sciences Research, 8(2):775-776. 2012.

44. Yousef, A.R.M., Mostafa E.A.M., and saleh. M.M.S., Response of olive seedlings to foliar sprays with amino acids and some micro elements. Agricultural and Biology Journal of North America. 2(7): 1108-1112. 2011.

45.Anonymous. Meteorology Region Department Records. Iran Weather Conditions 2011-2012. Tehran-Iran. 2012. www.weather.ir/.

46.Malakuti, M.J., Keshavarz, P. and Karimian, N., The way of distinction and better recommendation of fertilizer for sustainable agriculture. Publications of Tarbiat Modarres University. 2(7). 132-138. 2007.

47. MartinTanguy, J., Metabolism and function of polyamines in plants: recent development (new approaches). Plant growth Regul.34:135-48. 2001.
48.Smith, T.A., Polyamines. Ann. Rev. Plant Physiol., 36: 117-43. 1985.

49.Näsholm, T, Edfast, A, Ericsson, A. and Norden, L., Accumulation of amino acids in some boreal forest plants in response to increased nitrogen availability. New Phytol 126:137-143.1994.

50.Bauer, G.A., Bazzaz, F.A., Minocha, R, Long, S, Magill, A, Aber, J. and Berntson, G.M., Events of chronic N additions on tissue chemistry, photosynthetic capacity and carbon sequestration potential of a red pine (Pinus resinosa Ait.) stand in the NE United States. For Ecol Manag 196:173-186. 2004.

51.Bouché, N. and Fromm, H. GABA in plants: just a metabolite Trend. Plant Sci 9:110-115. 2004.

52.Dixon, R.A. and Paiva, N.L., Stress-induced phenylpropanoid metabolism. Plant Cell, 7: 1085-1097. 1995.

53.Grace, S.C. and Logan, B.A., Energy dissipation and radical scavenging by the plant phenylpropanoid pathway. Phil. Trans. Royal Soc. B, 355: 1499-1510. 2000.

54.Mottaghain, A., Pirdashti, H., Bahmanyar, M.A. and Abbasian, A., Leaf and seed micronutrient accumulation in soybean cultivars in response to integrated organic and chemical fertilizers application. Pakistan journal of biological sciences. 11(9):122733. 2008.

55.Saha, R., Palit, S. B., Ghosh, C. and Mittra, B.N., Performance of Aloe vera as Influenced by Organic and Inorganic Sources of Fertilizer Supplied through Fertigation. Acta Hort. 676. 171-175. 2005.

56.Youssef, E.A.E., Increasing drought tolerance of gladiolus plants through application of some growth regulators. M.Sc. Thesis, Fac. Agric., Zaggazig Univ., Egypt. 2007.

57.Ibrahim, M.E., Bekheta, M.A., El-Moursi, A. and Gafar, N.A. Improvement of growth and seed yield quality of Vicia faba L. plants as affected by application of some bioregulators. Aust. J. Basic and Appl. Sci., 1(4): 657-666. 2007.

58. Kowalzy, k. and Zielony, T., Effect of Aminoplant and Asahi on yield and quality of lettuce grown on rockwool. Conf. of bio stimulators in modern agriculture, Warsaw, Poland. 2008. 
